\title{
Stability of ${ }^{13} \mathrm{C}$-Urea Breath Test Samples Over Time in the Diagnosis of Helicobacter pylori
}

\author{
Tsachi Tsadok Perets, ${ }^{1,3 *}$ Einav Shporn, ${ }^{1}$ Doron Boltin, ${ }^{2,3}$ Ram Dickman, ${ }^{2,3}$ \\ and Yaron Niv ${ }^{2,3}$ \\ ${ }^{1}$ Gastroenterology Laboratory, Rabin Medical Center - Beilinson Hospital, Petah Tikva, Israel \\ ${ }^{2}$ Department of Gastroenterology, Rabin Medical Center - Beilinson Hospital, Petah Tikva, Israel \\ ${ }^{3}$ Sackler Faculty of Medicine, Tel Aviv University, Tel Aviv, Israel
}

\begin{abstract}
Background: The accuracy and repeatability of breath test in the diagnosis of Helicobacter pylori infection have not been adequately investigated. Although it has been shown that storage for long periods does not affect the analysis results, no data are available on the effect of repetitive testing. In this study, our aim was to evaluate the repeatability of the analyses of breath samples at room temperature. Methods: A total of 202 positive breath samples were collected in duplicates, before and after administration of $75 \mathrm{mg}{ }^{13} \mathrm{C}$ - urea dissolved in $100 \mathrm{ml}$ of orange juice. Breath test results were expressed as delta ${ }^{13} \mathrm{CO}_{2}$. The cut-off value was 3.5 parts per thousand. Each sample was analyzed in a mass spectrometer 7 ,

14,21 , and 28 days after collection. The accuracy calculation was based on the comparison of the delta ${ }^{13} \mathrm{CO}_{2}$ obtained in the three consecutive weeks following the first test run to the delta ${ }^{13} \mathrm{CO}_{2}$ obtained in the first test run. Results: Two hundred (99\%), $197(97.52 \%)$, and $196(97 \%)$ of the 202 samples tested positive in the second, third, and fourth test runs, respectively. The accuracy of the delta ${ }^{13} \mathrm{CO}_{2}$ was $98.6 \%, 99.2 \%$, and $96.7 \%$ in the three consecutive runs, respectively. Conclusion: Short-term storage of 1 month does not affect sample stability or the results of ${ }^{13} \mathrm{C}$-urea breath tests in up to three consecutive repeats. J. Clin. Lab. Anal. 30:231-234, 2016. (C) 2015 Wiley Periodicals, Inc.

Key words: ${ }^{13} \mathrm{C}$-urea breath test; reproducibility; test accuracy; $H$. pylori diagnosis; IRMS
\end{abstract}

\section{INTRODUCTION}

Helicobacter pylori (H. pylori) is a ubiquitous, flagellated gram-negative bacterium that infects approximately $50 \%$ of the world's population with an even higher prevalence in developing countries. Colonization of the gastric mucosa by $H$. pylori may cause chronic gastritis, which may advance to gastric and duodenal ulcers, mucosaassociated lymphoid tissue (MALT) lymphoma, and gastric adenocarcinoma $(1,2)$.

The exact route of transmission and subsequent infection of $H$. pylori in humans is incompletely understood. It has been suggested that human-to-human transmission is oral-oral, fecal-oral, or both, since H. pylori has been isolated from saliva, feces, gastroesophageal refluxate, and vomitus of infected subjects (3-5). It has been hypothesized that the oral-oral transmission is the primary route in developed countries and fecal-oral transmission (primarily via contaminated water sources) is more frequent in developing countries (6).
Diagnosis of $H$. pylori may be managed through invasive and noninvasive methods. Invasive methods include urease test, histology, and bacterial culture, and are based on endoscopy procedure in which biopsy is taken from the infected tissue. Noninvasive methods include serology, which is unreliable in confirming $H$. pylori eradication, due to the slow and unpredictable decline in antibody titer (7), stool antigen and the most reliable ${ }^{13} \mathrm{C}$-urea breath test (UBT) $(8,9)$. UBT is also considered a safe laboratory method for $H$. pylori diagnosis, since the use of ${ }^{14} \mathrm{C}$-labeled urea has been replaced with the ${ }^{13} \mathrm{C}$, which

\footnotetext{
*Correspondence to: Tsachi Tsadok Perets, Gastroenterology Laboratory, Department of Gastroenterology, Rabin Medical Center, 39 Jabotinsky Street, Petah Tikva 49100, Israel. E-mail: tzahipe1@clalit.org.il
}

Received 20 September 2014; Accepted 24 November 2014

DOI $10.1002 /$ jcla. 21841

Published online in Wiley Online Library (wileyonlinelibrary.com). 
TABLE 1. A Sample of the Test Results From Four Test Runs and the Calculation of the Delta and Accuracy of the UBTs

\begin{tabular}{|c|c|c|c|c|c|c|c|c|c|c|c|}
\hline Sample & $\begin{array}{l}\text { First } \\
\text { run }\end{array}$ & $\begin{array}{l}\text { Second } \\
\text { run }\end{array}$ & $\begin{array}{l}\text { Third } \\
\text { run }\end{array}$ & $\begin{array}{l}\text { Fourth } \\
\text { run }\end{array}$ & $\begin{array}{c}\text { Delta }{ }^{13} \mathrm{CO}_{2} \\
\text { First } \\
\text { run }\end{array}$ & $\begin{array}{c}\text { Delta }{ }^{13} \mathrm{CO}_{2} \\
\text { Second } \\
\text { run }\end{array}$ & $\begin{array}{c}\text { Delta }{ }^{13} \mathrm{CO}_{2} \\
\text { Third } \\
\text { run }\end{array}$ & $\begin{array}{c}\text { Delta }{ }^{13} \mathrm{CO}_{2} \\
\text { Fourth } \\
\text { run }\end{array}$ & $\begin{array}{l}\text { Accuracy } \\
\text { Second } \\
\text { run } \%\end{array}$ & $\begin{array}{l}\text { Accuracy } \\
\text { Third } \\
\text { run } \%\end{array}$ & $\begin{array}{c}\text { Accuracy } \\
\text { Fourth } \\
\text { run } \%\end{array}$ \\
\hline $\operatorname{A} 1(T=0)$ & -26.019 & -25.521 & -25.431 & -24.112 & & & & & & & \\
\hline $\mathrm{A} 2(T=30)$ & -7.316 & -7.132 & -7.091 & -5.559 & 18.703 & 18.389 & 18.34 & 18.553 & 98.32 & 98.06 & 99.20 \\
\hline
\end{tabular}

is a nonradioactive carbon isotope. The use of a stable, nonradioactive isotope allows for repetition of the test as required, and is safe even in children and pregnant women $(8,10)$. Moreover, UBT is also considered highly accurate, with sensitivity and specificity values approaching $100 \%$. Furthermore, UBT is a reliable means of assessing the success of $H$. pylori eradication $(6,10-12)$.

The interpretation of UBT is carried out using either isotope-ratio mass spectrometer (IRMS) or isotopeselective nondispersive infrared spectroscope (NDIRS) and it is based on the ability of $H$. pylori to hydrolyze the covalent link between urea and ${ }^{13} \mathrm{C}$, which is a unique feature of this pathogen (13). The accuracy and repeatability of UBT in the diagnosis of $H$. pylori infection have been a source for debate over recent years. Although it has been shown that storage for long periods does not affect the analysis results, no data are available on the effect of repetitive testing using tubes from the same sample (14-16).

Our laboratory provides UBT for $H$. pylori diagnosis to more than 4 million members of Clalit Health Services (CHS). CHS is the largest health insurer in Israel and the third-largest health maintenance organization in the world. Our laboratory receives samples from the entire country, with an annual turnover of more than 60,000 UBTs. The aim of the present study was to evaluate the repeatability of UBT analyses in our laboratory IRMS system.

\section{METHODS}

\section{Sample Acquisition}

Breath tests were conducted by dedicated nurses in community CHS clinics. Patients were given $75 \mathrm{mg}$ of ${ }^{13} \mathrm{C}$ labeled urea mixed with $100 \mathrm{ml}$ of orange juice. Breath samples were collected at two intervals - prior to urea administration (T0) and 30 min post urea administration (T30). The samples composed of the T0 and T30 collection tubes (Vacutainer, Becton, Dickinson and Company, $\mathrm{NJ})$ were sent to our laboratory for ${ }^{13} \mathrm{C}$ UBT analysis for diagnosis of $H$. pylori infection. The tubes were analyzed by IRMS instruments (AP2003, Analytical Precision, UK).

\section{Sample Analysis}

A random sample of 202 positive and 22 negative samples corresponding to 448 tubes were selected for analysis. The results are expressed as the difference between the two scores - delta over baseline. The cutoff $\frac{13 C}{12 C}$ at $\mathrm{T} 30-\mathrm{T} 0$ was 3.5 parts per thousand (PPT), according to referral laboratories and the manufacturer's instructions. Any increase above 3.5 PPT was considered H. pylori positive.

Each pair of test tubes was analyzed by the IRMS system 7 days after collection, and then at intervals of 7 days for an additional 3 weeks. The accuracy calculation was based on the comparison of the results of the delta ${ }^{13} \mathrm{CO}_{2}$ obtained in the three consecutive weeks following the first run to the delta ${ }^{13} \mathrm{CO}_{2}$ obtained in the first run. Samples were stored in our laboratory at room temperature between each measurement.

\section{Statistical Analysis}

The results of the delta ${ }^{13} \mathrm{CO}_{2}$ from the first test run were considered as the baseline for evaluation. For every test run, the delta was calculated for each sample (T30 - T0) in absolute numbers. The accuracy percentage was calculated by dividing each delta value from the second, third, and fourth test runs by the delta value of the first run and then multiplying the result by 100 (Table 1). The final accuracy values were obtained by calculating the mean of the total accuracy values for each of the samples in the same test run (Fig. 1, Table 2).

\section{RESULTS}

\section{Test Accuracy and Reproducibility}

Two hundred and two samples with delta value above 3.5 PPT and 22 samples with delta value equal to or lower than 3.5 PPT were selected for examination of reproducibility and test-retest accuracy. Of the 202 positive samples, 200 samples in the second test run were reproducible and the accuracy of the delta ${ }^{13} \mathrm{CO}_{2}$ measurement calculated was $98.6 \%$ when compared to the delta ${ }^{13} \mathrm{CO}_{2}$ of the first test run. In the third test run, 197 of the original 202 samples were reproducible and the accuracy was $99.2 \%$. In the fourth and final test run 196 of the 


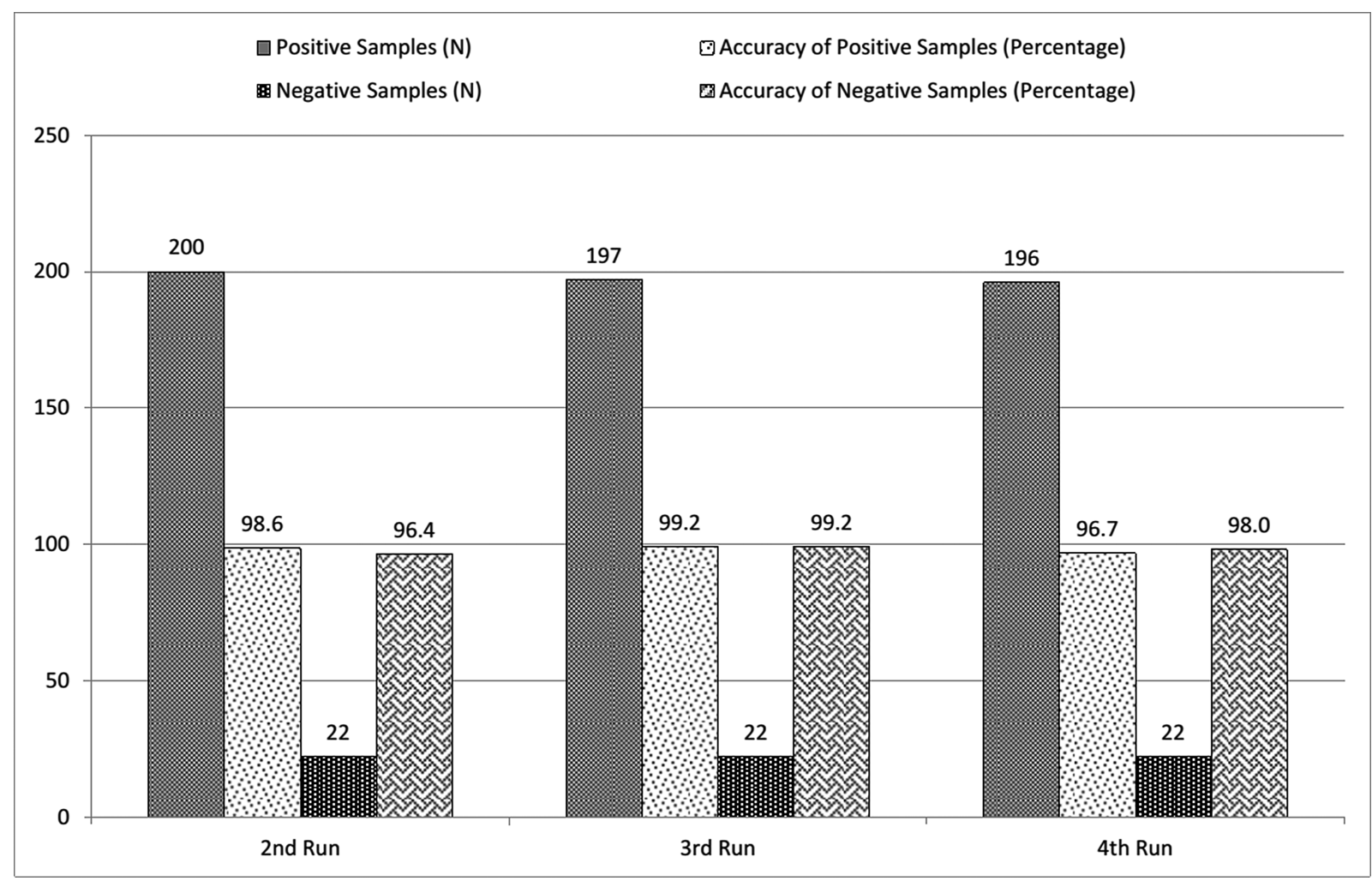

Fig. 1. Summary of accuracy values of three consecutive UBT runs over baseline. The delta ${ }^{13} \mathrm{CO}_{2}$ of each of the positive and negative samples in the three consecutive test runs was compared to the delta ${ }^{13} \mathrm{CO}_{2}$ of the first test run (baseline). The accuracy values represent the mean of the total accuracy values for each of the samples in the same test run.

TABLE 2. Reproducibility of Consecutive Testing of Paired UBT Samples

First run Second run Third run Fourth run

\begin{tabular}{lrccc}
\hline Total positive samples $(N)$ & 202 & 200 & 197 & 196 \\
Accuracy (\%) & 100 & 98.6 & 99.2 & 96.7 \\
Reproducibility (\%) & 100 & 99.0 & 97.5 & 97.0 \\
Total negative samples $(N)$ & 22 & 22 & 22 & 22 \\
Accuracy (\%) & 100 & 96.4 & 99.2 & 98.0 \\
Reproducibility (\%) & 100 & 100 & 100 & 100
\end{tabular}

original 202 samples were reproducible and the accuracy was $96.7 \%$ (Table 2, Fig. 1).

Of the 22 negative samples, 22 samples were reproducible in all three consecutive test runs post baseline. The accuracy values were $96.4 \%, 99.2 \%$, and $98.0 \%$ in the second, third, and fourth test runs, respectively. (Table 2, Fig. 1).

\section{Unreadable Samples}

Of the 202 positive samples collected, two samples became unreadable by the IRMS instrument after the first test run reducing the reproducibility percentage to $99 \%$. Of the remaining 200 samples, an additional three samples became unreadable after the third test run, reducing the reproducibility percentage to $97.5 \%$. Finally, after the fourth test run another single sample became unreadable, reducing the total reproducibility percentage to $97.0 \%$ (Table 2 , Fig. 1). Interestingly, all negative samples were readable during the three consecutive test runs post baseline, preserving a reproducibility rate of $100 \%$ (Table 2, Fig. 1).

\section{DISCUSSION}

H. pylori remains a widespread bacterium throughout the world, and therefore accurate diagnosis followed by a successful eradication of the bacterium is an important objective of health-care providers and patients alike (1-3). UBT is considered an accurate and noninvasive laboratory procedure $(8,9)$, and thus serves as the de facto gold standard for $H$. pylori diagnosis worldwide.

Consequently, $H$. pylori diagnosis in Israel is primarily achieved using UBTs that are sent to our laboratory for analysis in subjects prior to treatment and for confirmation of eradication. In contrast to other major laboratory systems, our high-yield IRMS instruments consume only 
a small portion of the exhaled $\mathrm{CO}_{2}$ in the sample tubes and, therefore, repetition of UBT on the same sample is feasible. In this aspect it is important to mention that NDIRS instruments, although quite accurate, are somewhat limited by their high consumption of gas in the UBT tubes. This allows for a maximum of two runs per sample (17).

Despite the fact that our measurements demonstrated high reproducibility values, five positive samples became unreadable by the IRMS instrument during the four consecutive test runs. We speculate that these samples had an insufficient concentration of exhaled $\mathrm{CO}_{2}$ due to improper completion of the breath test at the remote clinics in the community. It is possible that the patient did not exhale enough $\mathrm{CO}_{2}$ into either or both test tubes or they were not sealed properly. In fact, the only test-limiting variable is insufficient $\mathrm{CO}_{2}$ concentration in the samples test tubes, thus proper examination and test tube handling should be exercised.

In this study we have evaluated the repeatability of UBT analysis over a period of 1 month, using 7-day intervals between measurements. According to our findings, it is apparent that 1-month storage at room temperature does not affect the sample's stability and does not significantly affect the test's diagnostic accuracy. More importantly, storage for 1 month does not affect the repeatability of the test, at least in three consecutive repeats. In conclusion, the UBT is a valid laboratory method for $H$. pylori diagnosis and eradication evaluation and it has high testretest reliability.

\section{ACKNOWLEDGMENT}

The authors thank Mr. Zvika Shporn for his assistance in data processing.

\section{REFERENCES}

1. Wroblewski LE, Peek RM, Wilson KT. Helicobacter pylori and gastric cancer: Factors that modulate disease risk. Clin Microbiol Rev 2010;23:713-739.
2. Sibony M, Jones NL. Recent advances in Helicobacter pylori pathogenesis. Curr Opin Gastroenterol 2012;28:30-35.

3. Li C, Ha T, Ferguson DA Jr, et al. A newly developed PCR assay of $H$. pylori in gastric biopsy, saliva, and feces. Evidence of high prevalence of $H$. pylori in saliva supports oral transmission. Dig Dis Sci 1996;41:2142-2149.

4. Kabir S. Detection of Helicobacter pylori DNA in feces and saliva by polymerase chain reaction: A review. Helicobacter 2004;9:115123

5. Arboleda RN, Schneider BG, Bravo LE, et al. Use of the noninvasive entero-test in the detection of Helicobacter pylori in children in an endemic area in Colombia. J Pediatr Gastroenterol Nutr 2013;57:192-196.

6. Di Rienzo TA, D'Angelo G, Ojetti V, et al. 13C-Urea breath test for the diagnosis of Helicobacter pylori infection. Eur Rev Med Pharmacol Sci 2013;17(Suppl 2):51-58.

7. Savarino V, Vigneri S, Celle G. The $13 \mathrm{C}$ urea breath test in the diagnosis of Helicobacter pylori infection. Gut 1999;45(Suppl 1):I18 I22.

8. Gal E, Abuksis G, Fraser G, et al. 13C-Urea breath test for validation of eradication rate of Helicobacter pylori in an Israeli population. IMAJ 2003;5:98-100

9. Calvet X, Sánchez-Delgado J, Montserrat A, et al. Accuracy of diagnostic tests for Helicobacter pylori: A reappraisal. Clin Infect Dis 2009; 48:1385-1391.

10. Logan RP. Urea breath tests in the management of Helicobacter pylori infection. Gut 1998;43(suppl 1):S47-S50.

11. Hawtin PR. Serology and urea breath test in the diagnosis of $H$. pylori infection. Mol Biotechnol 1999;11:85-92.

12. Choi J, Kim CH, Kim D, et al. Prospective evaluation of a new stool antigen test for the detection of Helicobacter pylori, in comparison with histology, rapid urease test, (13)C-urea breath test, and serology. J Gastroenterol Hepatol 2011;26:1053-1059.

13. Savarino V, Mela GS, Zentilin P, et al. Comparison of isotope ratio mass spectrometry and nondispersive isotope-selective infrared spectroscopy for 13C-urea breath test. Am J Gastroenterol 1999;94:1203-1208.

14. Colaiocco Ferrante L, Papponetti M, Marcuccitti J, Neri M, Festi D. 13C-urea breath test for Helicobacter pylori infection: Stability of samples over time. Scand J Gastroenterol 1999;34:942-943.

15. Leodolter A, Domínguez-Muñoz JE, von Arnim U, Manes G, Malfertheiner P. 13C-urea breath test for the diagnosis of Helicobacter pylori infection. A further simplification for clinical practice. Scand J Gastroenterol 1998;33:267-270.

16. Peng NJ, Hsu PI, Lee SC, et al. A 15-minute [13C]-urea breath test for the diagnosis of Helicobacter pylori infection in patients with non-ulcer dyspepsia. J Gastroenterol Hepatol 2000;15(3):284-289.

17. Hilderbrand P, Beglinger C. Nondispersive infrared spectrometry: A new method for the detection of Helicobacter pylori infection with the ${ }^{13} \mathrm{C}$-urea breath test. Clin Infect Dis 1997;25:1003-1005. 